\title{
1. Hydrate Shell Growth Measured Using NMR
}

\author{
2 Agnes Haber, Masoumeh Akhfash, Charles K. Loh, Zachary M. Aman, Einar O. Fridjonsson, Eric F. May, \\ 3 and Michael L. Johns*
}

4 School of Mechanical and Chemical Engineering, The University of Western Australia, 35 Stirling Highway, Crawley, Western

5 Australia 6009, Australia

6 ABSTRACT: Benchtop nuclear magnetic resonance (NMR) pulsed field gradient 7 (PFG) and relaxation measurements were used to monitor the clathrate hydrate shell 8 growth occurring in water droplets dispersed in a continuous cyclopentane phase. 9 These techniques allowed the growth of hydrate inside the opaque exterior shell to be 10 monitored and, hence, information about the evolution of the shell's morphology to be 11 deduced. NMR Relaxation measurements were primarily used to monitor the hydrate 12 shell growth kinetics, while PFG NMR diffusion experiments were used to determine

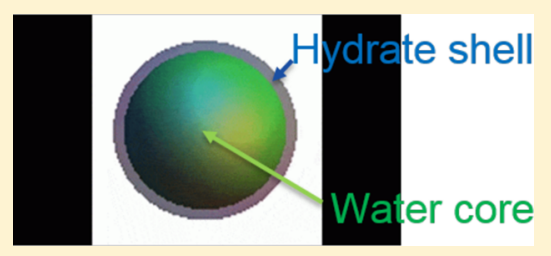
the nominal droplet size distribution (DSD) of the unconverted water inside the shell core. A comparison of mean droplet sizes obtained directly via PFG NMR and independently deduced from relaxation measurements showed that the assumption of the shell model-a perfect spherical core of unconverted water-for these hydrate droplet systems is correct, but only after approximately $24 \mathrm{~h}$ of shell growth. Initially, hydrate growth is faster and heat-transfer-limited, leading to porous shells with surface areas larger than that of spheres with equivalent volumes. Subsequently, the hydrate growth rate becomes mass-transferlimited, and the shells become thicker, spherical, and less porous.

\section{INTRODUCTION}

19 Clathrate hydrates (hereafter referred to as hydrates) are ice20 like crystalline compounds formed when hydrocarbon and 21 water molecules come into contact under high pressures and 22 low temperatures. ${ }^{1}$ Hydrates were discovered by Sir Humphry 23 Davy in $1810^{1}$ and for a long period remained a laboratory 24 curiosity. Nowadays hydrates are of interest across many 25 diverse fields such as $\mathrm{CO}_{2}$ sequestration, separation processes, 26 natural gas transportation, and cool storage applications. ${ }^{2}$ 27 However, they are also a major concern for the oil and gas 28 industry when they form and potentially block pipelines. ${ }^{3}$ 29 Maintaining flow in such pipelines is obviously essential to 30 production economics but also required to ensure safe 31 operations and minimize the risk of environmental pollution. ${ }^{4,5}$

32 In conventional oil transportation, where generally all three 33 phases are present (water, oil, and (natural) gas), hydrates may 34 form and agglomerate according to the conceptual model 35 (Figure 1) proposed by Turner et al. in collaboration with J. 36 Abrahamson. ${ }^{6,7}$ Initially, water droplets emulsify into the 37 continuous oil phase because of shear in the pipeline, often 38 assisted by other chemical agents in the oil phase. ${ }^{8}$ Hydrates 39 will nucleate and grow at the water interface on the droplet 40 surface, where there is also a supply of guest molecules 41 (typically methane) dissolved in the oil, to form a hydrate shell 42 around the droplet water core. Over time, the hydrate particles 43 will agglomerate and can eventually jam and plug the 44 pipeline. ${ }_{1,9,10}$ Flow assurance engineers seek to avoid this 45 situation by either preventing hydrate formation conditions 46 from occurring in the pipeline or, when this is unavoidable, by 47 actions based on a quantitative understanding of the 48 mechanisms by which hydrates grow and form plugs. 10

49 Generally, industry aims to completely prevent hydrate 50 formation by injecting sufficient quantities of inhibitor chemicals such as monoethylene glycol (MEG) or methanol; 51 however, such an approach becomes increasingly expensive the 52 deeper and further offshore the hydrocarbon exploration and 53 transportation occurs. ${ }^{11}$ Accordingly, industry is now moving 54 from complete hydrate avoidance to a risk management 55 approach (that may involve partial inhibition). ${ }^{4}$ However, 56 there are still significant knowledge gaps relating to how 57 hydrates form, agglomerate, and plug pipelines, as well as with 58 respect to the exact mechanisms by which various forms of 59 hydrate inhibition, that target these phenomena, operate. ${ }^{12,13} 60$ Studying these phenomena at a microscopic level, such as 61 measuring adhesion/repulsion forces between hydrate particles 62 and understanding the chemistry between inhibitors and 63 hydrates at the interfacial level, is central to ongoing research 64 efforts aimed at addressing these knowledge gaps (see, e.g., ref 65 9).

Experimental methods used for characterizing hydrate shell 67 growth around water droplets, as schematically shown in Figure 68 1 , include X-ray and neutron diffraction, ${ }^{14}$ Raman spectrosco- 69 py, $^{15}$ optical spectroscopy $^{16}$ and infrared spectroscopy. ${ }^{17} 70$ Conventionally hydrate shell growth kinetics have been studied 71 by monitoring the gas consumption in a pressure cell. Such 72 experiments, however, do not quantify the morphology of the 73 growth. ${ }^{18}$ Since the hydrate shell is visually opaque, most of the 74 previously mentioned techniques are limited in their ability to 75 quantitatively measure shell growth in detail, particularly with 76 respect to the shell interior. Alternative methods of probing 77 hydrate shell growth which, in principal, can measure the 78 morphology include differential scanning calorimetry (DSC) 79

Received: May 7, 2015

Revised: June 15, 2015 

Water entrainment
Hydrate growth
Agglomeration
Plugging

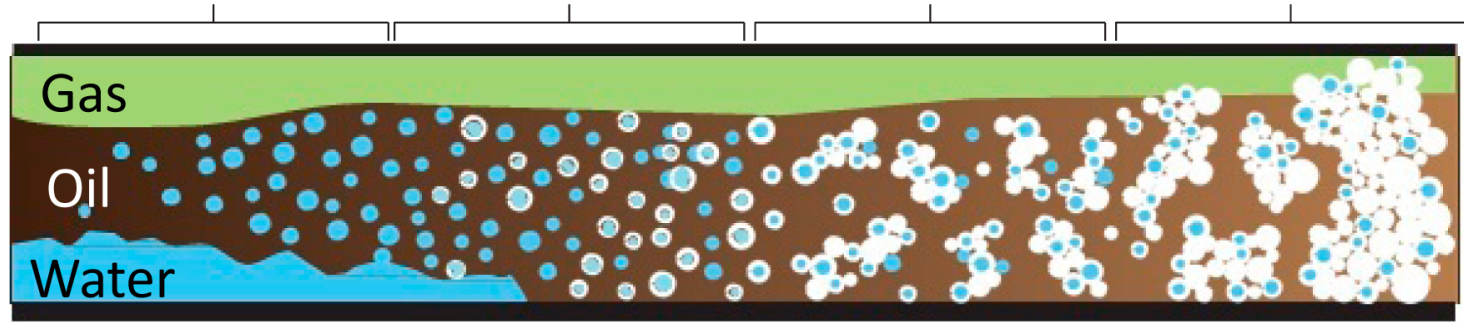

Figure 1. Conceptual picture of hydrate formation in an oil dominant multiphase flow line. Adapted with permission from ref 6 . Copyright 2006 Colorado School of Mines. Hydrate shell formation, indicated as white coloring, occurs around emulsified water droplets in the growth and agglomeration stages.

80 and nuclear magnetic resonance (NMR). ${ }^{19}$ The use of NMR 81 techniques to probe hydrate shell growth is the focus of the 82 work presented here.

83 NMR provides a noninvasive technique that does not require 84 optical access; it can be readily applied to determine droplet 85 size distributions in emulsions, ${ }^{20,21}$ the precursor micro86 structure to shell growth. This attribute has been used by 87 Aichele et al. ${ }^{19}$ to study subsequent hydrate growth within 88 water-in-oil emulsions in which hydrates were formed using 89 methane. The NMR methodology employed relied on the 90 quantification of restricted molecular water self-diffusion via the 91 application of NMR pulsed field gradient (PFG) techniques. 92 They applied these techniques to consider whether hydrate 93 formed via the shell model or via the complete conversion of 94 discrete droplets above a critical diameter (the threshold 95 model). Their data were shown to be consistent with the shell 96 model and in accordance with other evidence in the literature. ${ }^{1}$ 97 Restriction of (water) molecular self-diffusion within the 98 emulsion droplets is the physical phenomenon that enables 99 quantification of the droplet size distribution (DSD) in an 100 emulsion using PFG techniques. This restriction, however, can 101 result from the original emulsion droplet surface, or, as is the 102 case for the work presented here and by Aichele et al., ${ }^{19}$ the 103 inner surface of the hydrate shells. Thus, PFG NMR allows 104 probing of the inner water content of the hydrate encrusted 105 droplets.

106 A number of other relevant studies have sought to use NMR 107 techniques to measure hydrate formation. Gao et al. ${ }^{22}$ studied 108 the formation of hydrates in a THF and $\mathrm{D}_{2} \mathrm{O}$ system. As $\mathrm{D}_{2} \mathrm{O}$ 109 does not provide a ${ }^{1} \mathrm{H}$ NMR signal, they were able to 110 quantitatively monitor the disappearance of the THF signal 111 over time as it was converted into hydrate. Melnikov et al. ${ }^{23}$ 112 used ${ }^{1} \mathrm{H}$ NMR to study phase transformations of water in gas 113 hydrate systems including their metastable states at temper114 atures below the ice point for a Freon-water hydrate system. 115 Majid et al. ${ }^{24}$ used NMR relaxation methods to measure 116 emulsion droplet size distributions which were a precursor to 117 hydrate formation.

118 Much of our understanding about hydrate formation and 119 growth has been derived from visual measurements in optically 120 transparent systems. Chen et al. ${ }^{25}$ observed hydrate formation 121 on suspended gas bubbles in a counterflowing water column, 122 where mobile hydrate plates at the gas-water interface were 123 found to precede rapid growth of the hydrate shell. 124 Quantitative visual observations, collected over the past 2 125 decades, have generally investigated the formation of hydrate 126 films across flat horizontal interfaces between water and the 127 respective hydrocarbon. Hydrate film thickness and propaga- tion rate can be measured with different techniques, in 128 particular laser interferometry, micrometer measurements, and 129 visual microscopy. Taylor et al. ${ }^{26}$ presented visual measure- 130 ments of cyclopentane hydrate film thickness with the help of 131 digital video microscopy; this film thickness increased from 14132 to $34 \mu \mathrm{m}$ over a subcooling range of $4-10 \mathrm{~K}$. The film required 133 $200 \mathrm{~min}$ to reach a steady-state condition. Parallel data for 134 methane hydrate films, which increased in thickness from 18 to 135 $46 \mu \mathrm{m}$ over a subcooling range of $3.5-7.5 \mathrm{~K}$, were broadly 136 consistent with film thickness estimates of $10-52 \mu \mathrm{m}$ from 137 Makogon et al. ${ }^{27}$ and visual observation of $8-48 \mu \mathrm{m}$ hydrate 138 film growth on a suspended gas bubble by $\mathrm{Li}$ et al. ${ }^{28} \mathrm{Wu}$ et al. ${ }^{29} 139$ estimated hydrate film thicknesses of $1.3-25 \mu \mathrm{m}$ for a 140 methane-propane mixed gas hydrate system observed with a 141 HP-Video cell.

142

Following formation of this initial hydrate film (which in the 143 case of water emulsion droplets will be a hydrate shell), 144 continued hydrate growth is limited by mass transport of the 145 guest species and/or water across the hydrate shell lattice. ${ }^{7}$ This 146 continued hydrate growth is a critical parameter for estimating 147 the total hydrate fraction, residual water, and hence droplet 148 cohesion for any hydrate residence times extending to weeks; 149 for example during unplanned shut-ins (where production is 150 halted) of oil production lines. The current study represents the 151 first effort to quantify this continued hydrate growth with 152 NMR, a noninvasive technique that is not constrained by the 153 opaque hydrate shell on the emulsion droplets.

In this work, NMR was used to study the formation and 155 subsequent growth of cyclopentane hydrate films on water 156 droplets dispersed in (excess) cyclopentane. The kinetics of 157 water consumption as cyclopentane hydrate formed (and the 158 resultant hydrate shell grew on the water droplets) was 159 measured using two independent NMR signal relaxation 160 measurements under hydrate-forming conditions over several 161 weeks. Simultaneously the diameter of the water core within 162 the hydrate shell was measured using PFG NMR techniques. 163 Thus, we extend beyond the study of Aichele et al., ${ }^{19}$ that used 164 PFG NMR to confirm the formation of a hydrate shell on the 165 emulsion droplets, to quantify the evolution in the water core 166 diameter as hydrate film growth occurs. We are also able, for 167 the first time, to infer the water core diameter from the NMR 168 relaxation measurements. As detailed later, comparison of the 169 water core diameters as measured by PFG NMR and NMR 170 relaxation measurements respectively allows the morphological 171 assumption of a perfect sphere for the water core to be tested. 172 All NMR measurements were performed using a benchtop 173 comparatively low field and, hence, more readily accessible 174 NMR apparatus. 


\section{BACKGROUND}

176 2.1. Relevant NMR Theory. NMR relaxation refers to the 177 process through which all of the nuclear spins in a sample 178 return to thermodynamic equilibrium (the lowest energy 179 state $)^{30}$ following excitation. In NMR two major relaxation 180 processes can occur: longitudinal relaxation $\left(T_{1}\right)$ and transverse 181 relaxation $\left(T_{2}\right){ }^{31}$ The longitudinal relaxation time is the time 182 needed for the spins to realign with the axis of the external 183 magnetic field and give up all their energy to the lattice in order 184 to restore thermal equilibrium. To determine the $T_{1}$ relaxation 185 time, we used the saturation recovery method, which can be 186 described by

${ }_{187} S(t)=S_{\infty}\left(1-\exp \left(-t / T_{1}\right)\right)$

188 where $S$ is the signal measured at time $t$ and $S_{\infty}$ is the signal 189 intensity at equilibrium. The transverse relaxation time is the 190 time characteristic in which the spins dephase with respect to 191 one another due to molecular interactions or magnetic field 192 inhomogeneities. It can be described by

$\left.193 \quad S(t)=S_{0} \exp \left(-t / T_{2}\right)\right)$

194 where $S$ is the signal measured at $t$ and $S_{0}$ is the signal intensity 195 at time 0 . In the case of a porous environment, for example a 196 saturated rock sample, relaxation times for contained fluid 197 (usually water) give important information about porosity, 198 surface-to-volume ratio, permeability, formation factor, tortuos199 ity, pore size distribution, and pore connectivity. ${ }^{32}$ This is 200 possible because the relaxation times are sensitive to both 201 molecular motion and pore geometry. Additional information 202 can also be extracted by performing such relaxation measure203 ments as a function of resonance frequency using fast field 204 cycling techniques. ${ }^{33}$ For a porous medium the dimensions of 205 the pores are related to relaxation times by the Brownstein206 Tarr equation. ${ }^{34,35}$ The $T_{1}$ and $T_{2}$ relaxation times of the 207 contained fluid can be written as

$$
\begin{aligned}
& \frac{1}{T_{1}}=\frac{1}{T_{1, \text { bulk }}}+\rho_{1} \frac{S}{V} \\
& \frac{1}{T_{2}}=\frac{1}{T_{2, \text { bulk }}}+\rho_{2} \frac{S}{\mathrm{~V}}+\frac{D}{12}\left(\gamma G t_{\mathrm{E}}\right)^{2}
\end{aligned}
$$

208 where $T_{1}$ and $T_{2}$ are the apparent relaxation times $(\mathrm{s}), T_{1, \text { bulk }}$ 209 and $T_{2, \text { bulk }}$ are the relaxation times in a nonrestricted 210 environment $(\mathrm{s}), \rho_{1}$ and $\rho_{2}$ are the surface relaxivities $(\mu \mathrm{m} /$ $211 \mathrm{~s}), S / V$ is the surface-to-volume ratio $\left(\mathrm{m}^{-1}\right)$ of the confining 212 medium, $D$ is the diffusion coefficient $\left(\mathrm{m}^{2} / \mathrm{s}\right)$ of the contained 213 fluid, $\gamma$ is the gyromagnetic ratio $(\mathrm{MHz} / \mathrm{T}), G$ is the 214 background magnetic field gradient strength $(\mathrm{T} / \mathrm{m})$, and $t_{\mathrm{E}}$ is 215 the echo time (s). The Brownstein-Tarr theory equations (eq $2162)$ are strictly only valid for the fast diffusion limit $(\rho a / D \ll 1)$, 217 this was found to be true for the vast majority of the acquired 218 data with $(\rho a / D)$ always $<1$. If $t_{\mathrm{E}}$ is sufficiently short, then the 219 third right-hand-side term of eq $2 b$ can be neglected and the 220 relaxation is dominated by just surface relaxation. In this case, 221 the relaxation rate of a fluid in a pore is given by ${ }^{36}$

$$
{ }_{222} \quad \frac{1}{T_{1,2}}=\frac{1}{T_{1,2, \text { bulk }}}+\rho_{1,2} \frac{S}{V}
$$

223 In the cases when the confining geometry of the fluid (the 224 pore) is a perfect isolated sphere, such as an emulsion droplet, $225 S / V=3 / a$ where $a$ is the sphere radius. Probability 226 distributions of $T_{1}$ or $T_{2}$ were determined using standard regularization techniques, the specific details of which are 227 provided by Mitchell and Chandrasekera. ${ }^{37} 228$

The measurement of unrestricted self-diffusion of molecules 229 in a liquid by PFG NMR methods was first demonstrated by 230 Stejskal and Tanner. ${ }^{38}$ The method essentially relies on signal 231 attenuation due to random motion (diffusion) of the molecules 232 between two imposed magnetic field gradients. The resultant 233 NMR signal loss $\left(I=S / S_{0}\right)$ is described by

$$
I=\exp \left(-D(\gamma \delta g)^{2}\left(\Delta-\frac{\delta}{3}\right)\right)
$$

where $D$ is the self-diffusion coefficient of the relevant 236 molecules and $\Delta, \delta$, and $g$ are the temporal duration between 237 application of the two magnetic field gradients, the duration of 238 a single magnetic field gradient application, and the strength of 239 the magnetic field gradient, respectively. $\gamma$ is the gyromagnetic 240 ratio of the spin; the ${ }^{1} \mathrm{H}$ nucleus, which has $\gamma=2.68 \times 10^{8} T^{-1} 241$ $s^{-1}$, was used exclusively in the work presented here. $I$ is thus 242 measured as a function of $g$, $\delta$, or $\Delta$ enabling the extraction of $D 243$ via application of eq 4 . The NMR pulse sequence most 244 commonly employed to achieve this with respect to emulsion 245 characterization is based on a stimulated echo signal, which will 246 be detailed in Materials and Methods.

247

In the case of restricted self-diffusion inside a spherical 248 geometry (e.g., an emulsion droplet, or in the hydrate water 249 core), the NMR signal attenuation can be approximated as ${ }^{39} 250$

$$
\begin{aligned}
& \ln I(D, a, g, \delta)=-2 \gamma^{2} g^{2} \sum_{m=1}^{\infty} \frac{1}{\alpha_{m}{ }^{2}\left(\alpha_{m}{ }^{2} a^{2}-2\right)}\left[\frac{2 \delta}{\alpha_{m}{ }^{2} D}-\frac{\psi}{\left(\alpha_{m}{ }^{2} D\right)^{2}}\right] \\
& \psi=2+\exp ^{-\alpha_{m}{ }^{2} D(\Delta-\delta)}-2 \exp ^{-\alpha_{m}{ }^{2} D \Delta}-2 \exp ^{-\alpha_{m}{ }^{2} D \delta}+\exp ^{-\alpha_{m}{ }^{2} D(\Delta+\delta)}
\end{aligned}
$$

where $\alpha_{m}$ is the $m$ th positive root of the equation:

$$
J_{5 / 2}(\alpha \alpha)-\frac{1}{\alpha \alpha} J_{3 / 2}(\alpha \alpha)=0
$$

$J_{k}$ is the Bessel function of the first kind of order $k$, and $a$ is the 255 droplet radius. Equations 5 assume that the NMR signal phase 256 $(\varphi)$ distribution is a Gaussian shape. An alternative to eqs 5257 exists in which it is assumed that the duration of the applied 258 magnetic field gradient pulse, $\delta$, is infinitely short. ${ }^{40}$ More 259 recently, however, $^{41}$ it was demonstrated that the use of the 260 block gradient pulse (bgp) approximation method to describe 261 restricted diffusion inside spherical droplets (emulsion droplet 262 sizing) offered improved accuracy compared to the assumption 263 of a Gaussian phase distribution or an infinitely short $\delta$ over all 264 practical considerations of experimental parameter space. The 265 bgp method is based on the generalized gradient waveform set 266 of methods, ${ }^{42-44}$ in which an eigenfunction expansion is used 267 to solve the Bloch Torrey equation in the presence of a 268 piecewise-constant gradient waveform. ${ }^{45}$ The Bloch Torrey 269 equation is reposed, for self-diffusion in a spherical emulsion 270 geometry, in (Laplace operator eigenbasis) matrix form as 271

$$
S(\tilde{t})=\mathbf{v}^{\dagger}\left(\prod_{k=1}^{N_{\mathrm{e}}} \mathrm{e}^{\left[\left(-\boldsymbol{\Lambda}+i\left(\tilde{\gamma}_{k} \cdot \hat{z}\right) \mathbf{Z}\right) \tilde{t}_{k}\right]}\right) \mathbf{v}
$$

where for reflective emulsion boundary with no surface 273 relaxation $v_{j}=\delta_{j 1}, \tilde{t}=\mathrm{Dt} / \mathrm{a}^{2}, \tilde{\gamma}=-c \gamma g a^{3}$, and $\Lambda_{j k}=\alpha_{n m}{ }^{2} \delta_{j k}, 274$ while $\mathbf{Z}$ for a sphere is given analytically by Grebenkov. ${ }^{45}$ Here 275 $\delta$ is the Kronecker delta. Note that $\boldsymbol{\Lambda}$ and $\mathbf{Z}$ respectively 276 represent the evolution of magnetization due to diffusion and 277 
278 gradient dephasing. Expansion of eq 6 for the stimulated echo 279 PFG pulse sequence used here allows the NMR signal 280 attenuation $(I)$ to be expressed as

281

$$
I=\frac{\mathbf{v}^{\dagger} \mathrm{e}^{(-\boldsymbol{\Lambda}+i(\tilde{\gamma} \cdot \hat{z}) \mathbf{Z}) \delta} \mathrm{e}^{-\boldsymbol{\Lambda}(\Delta-\delta)} \mathrm{e}^{(-\boldsymbol{\Lambda}+i(\tilde{\gamma} \cdot \hat{z}) \mathbf{Z}) \delta} \mathbf{v}}{\mathbf{v}^{\dagger} \mathrm{e}^{-\boldsymbol{\Lambda}(\Delta+\delta)} \mathbf{v}}
$$

282 where the only free parameter is the droplet size $a$. Greater 283 details regarding the mathematical derivation can be sourced 284 from Grebenkov ${ }^{46}$ and our implementation for emulsion 285 droplet sizing, as was exclusively employed in the work 286 presented here, is detailed in Lingwood et al. ${ }^{41}$

287 Most emulsions consist of a distribution of droplet radii, $P(a)$ 288 (number based), meaning the measured signal is described by 289 the following integral:

290

$$
I(D, g, \delta)=\frac{\int_{\infty}^{0} a^{3} P(a) I(D, a, g, \delta) \mathrm{d} a}{\int_{\infty}^{0} a^{3} P(a) \mathrm{d} a}
$$

291 where $I(D, a, g, \delta)$ is the signal attenuation function described by $292 \mathrm{eq} \mathrm{7.} \mathrm{The} \mathrm{extraction} \mathrm{of} P(a)$ from eq 8 is both an ill293 conditioned matrix inversion (when tackled analytically) and an 294 unstable minimization problem (when tackled numerically). 295 Previously we have demonstrated ${ }^{47}$ the use of the Tikinov 296 regularization method to numerically invert eq 6 with 297 generalized cross-validation (GCV) used for regularization 298 parameter selection; this does not require any assumption 299 regarding the droplet size distribution shape.

300 2.2. Relevant Hydrate Theory. Clathrate hydrates are 301 crystalline inclusion compounds, where molecular water cages 302 surround lighter hydrocarbon species. ${ }^{1}$ Two different cubic 303 hydrate structures are commonly found: I and II. Structure I 304 hosts smaller guest molecules and is the structure found most 305 commonly in the natural environment, whereas structure II 306 contains cages that can host larger guest molecules and is 307 predominantly found in oil and gas production systems. ${ }^{48}$ This 308 investigation used cyclopentane as an ambient pressure 309 structure II former that is immiscible with water. Consequently, 310 cyclopentane hydrate formation is generally confined to 311 interfaces between hydrocarbon and aqueous phases, which 312 together with the fact that it forms structure II, makes it a closer 313 analogue to the hydrates that form in oil and gas pipelines (as 314 opposed to THF which is often used in ambient pressure 315 hydrate studies). Structure II cyclopentane hydrates contain 16 316 small $5^{12}$ cavities (pentagonal dodecahedron) and 8 large $5^{12} 6^{4}$ 317 cavities (hexakaidecahedral), with 136 water molecules per unit 318 cell. $^{48}$ Cyclopentane hydrate has a relatively high hydrate319 forming equilibrium temperature of $7.7{ }^{\circ} \mathrm{C}$ at atmospheric 320 pressure. $^{49,50}$

321 Hydrate formation, in general, occurs in two steps: (1) 322 nucleation and (2) growth. Although hydrate growth occurs at 323 any temperature below the hydrate equilibrium temperature, 324 operating at such thermodynamically favorable conditions will 325 not guarantee the instantaneous nucleation of hydrates. ${ }^{26,51,52}$ 326 Nucleation time $\left(t_{\text {nuc }}\right)$ is the time interval between the 327 establishment of hydrate stability conditions and the formation 328 of the first hydrate clusters. In literature, there is a large 329 variability in $t_{\text {nuc; }}{ }^{53}$ Sloan and collaborators ${ }^{54}$ note that this is an 330 indication that $t_{\text {nuc }}$ is dictated by a stochastic process, 331 particularly for smaller sample volumes and lower subcool332 ings. ${ }^{55}$ Furthermore, the "type" of water employed for hydrate 333 formation in a static system (without flow) was also found to 334 play an important role. ${ }^{56}$ Thawed ice produced the shortest nucleation time. Numerous studies have attributed this 335 observation to the fact that the ice-hydrocarbon-aqueous 336 interface between phases acts as a heterogeneous nucleation 337 site. ${ }^{1,55,57,58}$ The incipient water from melted ice still contains 338 residual structure that it is hypothesized to provide a template 339 to form the water cage structure of a hydrate. ${ }^{1}$ If the study of 340 hydrate growth is the main objective, then promoting hydrate 341 nucleation is advantageous and, therefore, the use of recently 342 thawed ice (as is the case for experiments performed in the 343 work presented here) is convenient.

Following nucleation of hydrate at the interface between two 345 immiscible phases, hydrate film growth occurs at a rate that is 346 set initially by heat-transfer limitations. Visual methods have 347 been used to study the nature and morphology of this growth 348 along planar interfaces and across the exterior surface of 349 suspended droplets or bubble until a complete shell is formed, 350 after which time the amount of information extractable 351 becomes limited. Using data measured by Uchida et al., ${ }^{59} 352$ Mori proposed a model for hydrate film growth which related 353 the product of the initial film's lateral growth rate and the 354 thickness to the enthalpy of formation, a convective heat- 355 transfer coefficient and a power law dependency on the system 356 subcooling. ${ }^{60}$ This applicability of this heat-transfer-limited 357 growth model to the initial growth of planar hydrate films was 358 validated in 2007 by the experiments of Taylor et al. ${ }^{26}$ who 359 quantified both the film propagation rate and thickness as a 360 function of time for both cyclopentane and methane hydrates. 361 While the initial film thicknesses were about $12 \mu \mathrm{m}$ for 362 cyclopentane hydrates, these increased to final values of about 363 $25 \mu \mathrm{m}$ for subcoolings around $8 \mathrm{~K}$ over a period of $200 \mathrm{~min} .364$ For methane hydrates at this subcooling, the steady-state 365 thicknesses reported by Taylor et al. ${ }^{26}$ were $50 \mu \mathrm{m}$, which was 366 consistent with the results of other measurements of planar 367 interfaces. $^{27,28}$ However, as pointed out by Ohmura et al., ${ }^{61}$ the 368 initial phase of hydrate film growth is sensitive to the system 369 geometry (e.g., shell vs film) because this affects the nature of 370 the free-convective motion of the water sweeping the growing 371 hydrate film and determining the magnitude of heat transfer 372 possible.

Genov et al. ${ }^{62}$ proposed that the initial phase of rapid growth 374 gave rise to a porous hydrate shell that would subsequently 375 allow for reasonable rates of mass and heat exchange between 376 the phases. Chen et al. ${ }^{63}$ visually observed the formation of a 377 porous hydrate shell at the methane-water interface in a high- 378 pressure water tunnel, similarly concluding that the shell did 379 not introduce a significant barrier to mass transport between 380 the continuous phases in the early stages of growth. Following 381 the initial rapid phase of heat-transfer-limited growth, which 382 lasted about $10 \mathrm{~min}$, Taylor et al. ${ }^{26}$ observed growth occurring 383 at a much slower rate as the cyclopentane hydrate film 384 thickened to its steady-state value over several hours. This 385 second phase growth is mass-transfer-controlled and depends 386 not only on the diffusivity of the hydrocarbon molecules 387 through the film but also on the porosity of the hydrate film. ${ }^{64} 388$ Rao et al. ${ }^{65}$ studied the deposition and porosity of a methane 389 hydrate film on a cold steel surface, reporting porosity values 390 above $75 \%$ for the first $20 \mathrm{~h}$ of growth; after $75 \mathrm{~h}$ of annealing, 391 however, the film porosity decreased to approximately 5\%. For 392 spherical interfaces, as the hydrate shell grows thicker and/or its 393 porosity is reduced, mass-transfer resistance also increases and 394 the growth rate of the hydrate shell layer consequently will 395 decrease. This effect is consistently observed across many 396 studies. $^{18,66-69}$ Extrapolating from their measurements of 397 


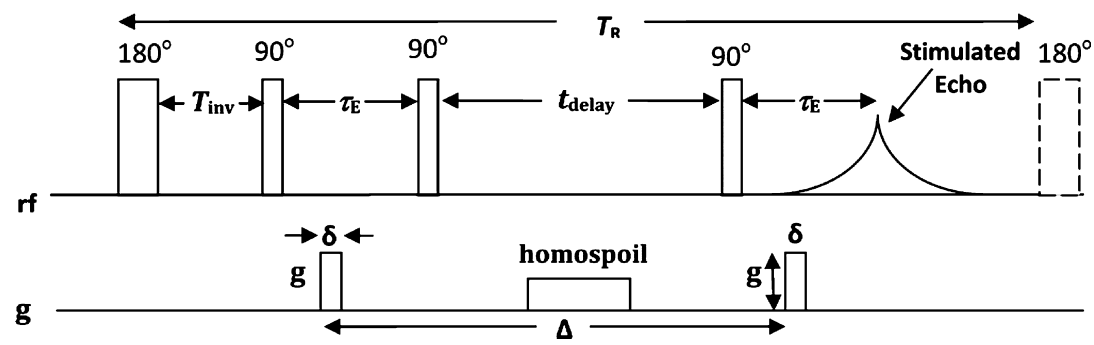

Figure 2. Modified SSE PFG pulse sequence which is able to differentiate between the water and the oil (cyclopentane) signal based on adequate differences in the $T_{1}$ relaxation parameter. This was enabled by dissolving a Gd complex in the cyclopentane prior to use.
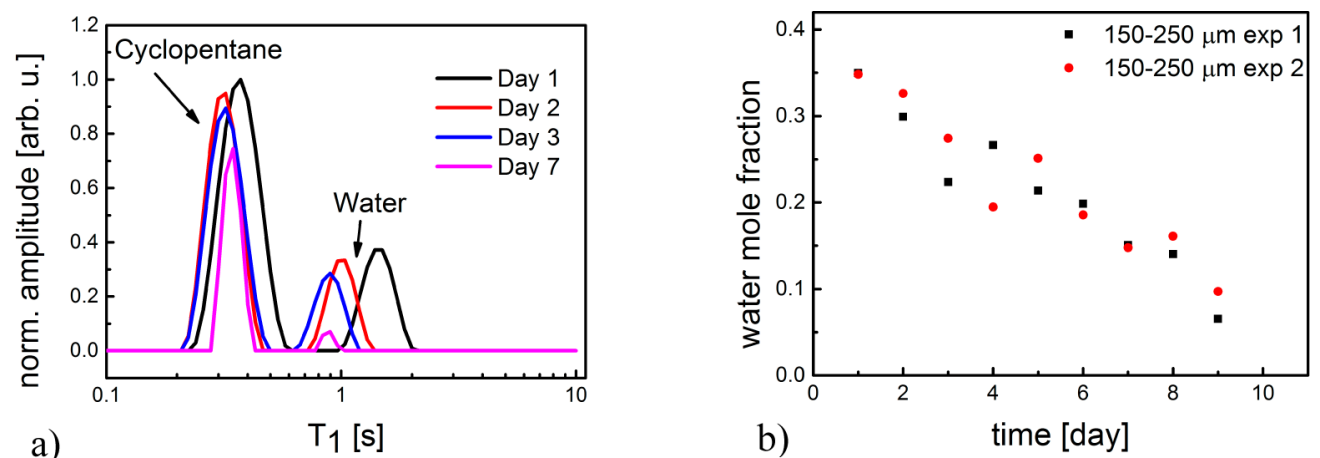

Figure 3. Results of the $T_{1}$ relaxation experiment: (a) $T_{1}$ distribution curves, showing the two sets of peaks for cyclopentane and water, respectively; (b) water mole fraction obtained for two independent experiments showing the reduction in water mole fraction as a thicker hydrate shell is formed.

398 planar hydrate films, Taylor et al. ${ }^{26}$ hypothesized that full 399 conversion of water contained within a spherical hydrate shell 400 might require time scales on the order of tens of hours.

\section{METHODOLOGY}

401 3.1. Materials and Methods. To prepare cyclopentane hydrates, 402 sieved ice particles (made from deionized water) with nominal 403 diameters between 150 and $250 \mu \mathrm{m}$ were mixed with excess 404 cyclopentane at $-20{ }^{\circ} \mathrm{C}$. A reduced selection of experiments was 405 also performed with ice particle nominal diameters between 75 and $406150 \mu \mathrm{m}$ and below $75 \mu \mathrm{m}$, respectively. As discussed earlier, the use of 407 thawed ice promotes a higher degree of initial molecular water 408 ordering and contributes to faster hydrate growth. ${ }^{55}$ The required 409 sieving of pulverized ice was performed in an appropriately cold 410 environment (the sieves being cooled with liquid nitrogen), after 411 which both the ice particle and the cyclopentane were separately 412 stored at $-20{ }^{\circ} \mathrm{C}$ using a Haake Phoenix cooling bath. The 413 cyclopentane and the ice particles were rapidly mixed in precooled 9 $414 \mathrm{~mm}$ inner diameter NMR glass test tubes that were subsequently 415 closed, then heated to $1{ }^{\circ} \mathrm{C}$, and maintained at room pressure. This 416 storage temperature was above the melting temperature of ice but 417 below the cyclopentane equilibrium temperature of $7.7^{\circ} \mathrm{C}$ at $1 \mathrm{bar}$, 418 which meant a subcooling of $6.7^{\circ} \mathrm{C}$. The NMR test tubes were then 419 placed in the benchtop NMR spectrometer (detailed later) in a $1{ }^{\circ} \mathrm{C}$ 420 environment; relaxation and diffusion experiments (also detailed later) 421 were performed daily for a period of at least 14 days. In this manner 422 we were able to monitor the gradual hydrate shell thickening following 423 initial rapid hydrate shell formation.

424 All NMR measurements were performed using a commercially 425 available benchtop NMR Minispec mq Bruker spectrometer, with a ${ }^{1} \mathrm{H}$ 426 resonance frequency of $20 \mathrm{MHz} .^{70}$ The Bruker Minispec features a 427 strong pulsed gradient $(4 \mathrm{~T} / \mathrm{m})$ for PFG purposes, is able to 428 accommodate $10 \mathrm{~mm}$ (outer diameter) NMR tubes, and provides 429 sample temperature control which was kept constant at $1{ }^{\circ} \mathrm{C}$ during all 430 experiments. For the NMR relaxometry measurements, standard 431 saturation recovery and $\mathrm{CPMG}^{71,72}$ pulse sequences were employed to 432 obtain the $T_{1}$ and $T_{2}$ relaxation time distributions, respectively.
The NMR pulse sequence used for all self-diffusion measurements 433 was the modified stimulated spin echo (SSE) PFG, as shown in Figure $434 \mathrm{f}_{2}$ 2. Since the Bruker Minispec has no spectral resolution, it is not $435 \mathrm{f} 2$ possible to directly differentiate the NMR signal from the water and 436 cyclopentane phases, respectively. Consequently a $180^{\circ}$ radio 437 frequency (rf) pulse is conventionally inserted at the pulse sequence 438 initiation, followed by a time delay equal to the null point of the oil 439 signal $\left(0.693 T_{1, \text { oil }}\right)$. This pulse sequence has previously been employed 440 to determine the size distribution of water-in-crude oil emulsions 441 employing the same NMR hardware. ${ }^{73}$ The relaxation times (both $T_{1} 442$ and $T_{2}$ ) for cyclopentane and water are, however, very similar (the $T_{1} 443$ at $1{ }^{\circ} \mathrm{C}$ for water is $1.5 \mathrm{~s}$; for cyclopentane it is $1.8 \mathrm{~s}$ ); thus selective 444 suppression-nulling of the cyclopentane signal using the SSE PFG 445 pulse sequence is not possible. Hence, in order to reduce the relaxation 446 time of the cyclopentane, we employed a paramagnetic gadolinium 447 complex that was soluble only in the cyclopentane. This complex was 448 gadolinium tetramethyl heptanedionate (Gd(III) TMHD), which was 449 purchased from Multivalent Laboratory. It is not soluble in water but 450 presents a solubility of $2.9 \mathrm{mmol} / \mathrm{L}$ in hexane ${ }^{74}$ and a similar solubility 451 in cyclopentane. Such paramagnetic compounds are generally used in 452 MRI as contrast agents to better differentiate between tissues. ${ }^{75}$ In our 453 case, we dissolved $0.6 \%(\mathrm{w} / \mathrm{w})$ Gd(III) TMHD salt into the 454 cyclopentane prior to mixing of the cyclopentane and any ice; the 455 Gd complex remained in solution at all temperatures considered. This 456 served to reduce the $T_{1}$ relaxation time for the cyclopentane from 1.8457 to $0.35 \mathrm{~s}$, enabling ready differentiation of the water and cyclopentane 458 contributions to acquired relaxation distributions, as well as effective 459 suppression-nulling of the cyclopentane NMR signal during PFG 460 measurements.

With reference to Figure 2, the observation time $(\Delta)$ used in all 462 experiments conducted here was $180 \mathrm{~ms}$, the gradient pulse length $(\delta) 463$ was $2 \mathrm{~ms}$, and the gradient strength $(g)$ was increased from 0 to $0.2 \mathrm{~T} / 464$ $\mathrm{m}$ in 16 increments. Inversion of the acquired data to produce the 465 emulsion droplet size distributions was performed using eq 8 and the 466 bgp method detailed earlier. 

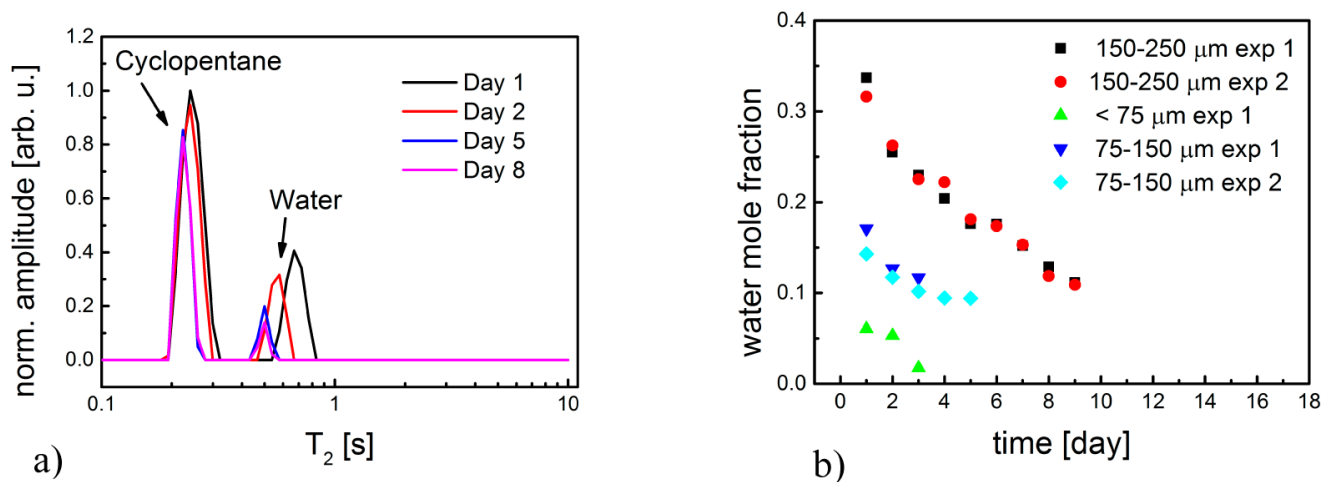

Figure 4. Results of the $T_{2}$ relaxation experiment for ice particles of nominal diameter 150-250 $\mu \mathrm{m}$ : (a) $T_{2}$ distribution curves, showing the two separate group of peaks for cyclopentane and water, respectively; (b) water mole fraction calculated by integrating the relaxation peaks for water and cyclopentane over time and adjusting for ${ }^{1} \mathrm{H}$ density for three different ice particle sizes.
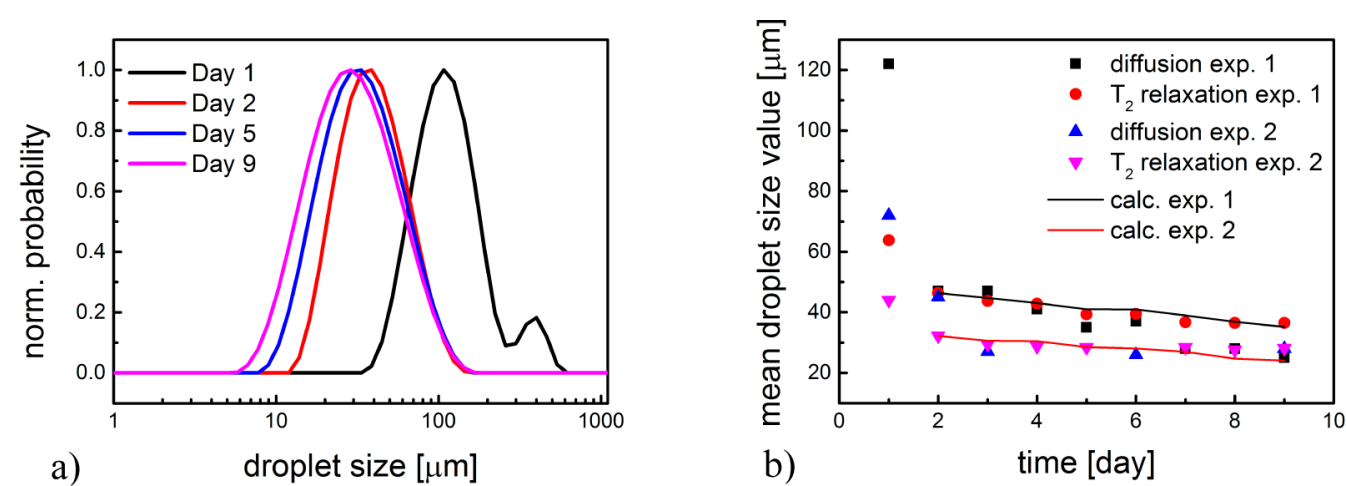

Figure 5. (a) Droplet size distribution (DSD) obtained with the PFG NMR diffusion experiments. A decrease in the droplet mean value is observed over 9 days, corresponding to the shrinking of the water core. (b) Comparison between the mean droplet size obtained by self-diffusion and $T_{2}$ relaxation measurements respectively for two independent experiments. The solid lines are the predicted change in droplet size from day 2 onward, based on the residual water content measured and reported in Figure 4b, using the diameter, as measured using relaxation measurements, on day 2 as an anchor point.

\section{RESULTS AND DISCUSSION}

468 4.1. Hydrate Growth Kinetics. A set of sample $T_{1}$ 469 relaxation data is presented in Figure 3a as a function of time 470 following thawing of the ice content in the sample at $1{ }^{\circ} \mathrm{C}$, as 471 detailed previously. The $T_{1}$ value for water is $\sim 1.5 \mathrm{~s}$, which 472 corresponds to the right group of peaks in Figure $3 \mathrm{a}$, and the $T_{1}$ 473 value for cyclopentane is $\sim 350 \mathrm{~ms}$, which corresponds to the 474 left group of peaks in the same figure. The addition of the Gd 475 complex clearly facilitates the quantitative differentiation of the 476 cyclopentane and water contribution in these relaxation 477 distributions. Over a period of 7 days, the water peak shifted 478 to the left and diminished in intensity, while there was 479 comparatively little change in the cyclopentane peak. The shift 480 to lower values for the water peak reflects the fact that water481 occupied domains decreased in size consistent with eq 3; the 482 reduction in intensity reflects the reduction in the liquid water 483 content of the sample as it converted to hydrate, which is NMR 484 invisible for these measurements. The small reduction in the 485 cyclopentane reflects changes in the cyclopentane content in 486 the detection field of view as well as by consumption to form 487 hydrate. It is important to note, however, that the cyclopentane 488 is in excess and that $13.5 \mathrm{~mol}$ of water are consumed per mole 489 of cyclopentane due to selective guest occupancy in the large 490 cage of structure $\mathrm{II}^{1}{ }^{1}$

491 Using the areas under the peaks and correcting for 492 differences in the relative ${ }^{1} \mathrm{H}$ density between water and 493 cyclopentane, it is possible to calculate the water mole fraction in the detected sample volume-its evolution with time 494 following ice thawing at $1{ }^{\circ} \mathrm{C}$ is presented in Figure 3b. An 495 independent repeat experiment is also shown indicating the 496 reproducibility of the observed hydrate growth kinetics. The 497 "noise-like" variation evident in the data in Figure 3b is a 498 combined consequence of noise in the acquired NMR data and 499 the required numerical data inversion employing the 500 regularization techniques in eq 8 to produce the probability 501 distributions presented in Figure $3 \mathrm{a}$.

The corresponding $T_{2}$ relaxation time distribution data for 503 the preceding experiments are shown in Figure $4 \mathrm{a}$, along with $504 \mathrm{f4}$ the resultant water mole fractions in Figure $4 \mathrm{~b}$ determined as a 505 function of time following ice thawing. The water and 506 cyclopentane peak groups are distinct in Figure 4a, and their 507 general behavior is consistent with that observed based on $T_{1} 508$ measurements (in Figure 3). Also shown in Figure $4 \mathrm{~b}$ are the 509 data corresponding to reduced ice particle diameters of 75-150 510 and $<75 \mu \mathrm{m}$. The hydrate formation kinetics (shown in Figure 511 $4 \mathrm{~b})$ are very consistent with that shown in Figure $3 \mathrm{~b}$. The 512 acquired NMR data are considerably less noisy than those used 513 to construct Figure 3 and hence has higher reproducibility as 514 revealed by the independent experiment also shown in Figure 515 4b. Since $T_{2}$ measurements can be performed more rapidly, 516 which allows for more signal averaging, they will exclusively be 517 used in the future for such hydrate growth kinetic measure- 518 ments. Also evident in Figure 4b is a distinct change in hydrate 519 formation rate between day 1 and day 2 . This we speculate is a 520 
521 consequence of a transition from heat-transfer-limited growth 522 to mass-transfer-limited growth, for which there is considerable 523 literature evidence, as discussed earlier. Because the initial ice 524 particles size is reduced, there is a reduction in the residual 525 water content on day 1 . As discussed later, this is consistent 526 with the initial rapid formation of a hydrate film; assuming a 527 constant thickness for such a film would reduce the residual 528 water content as ice particle size is reduced. Subsequent kinetics 529 from day 2 onward do not show any obvious trend with initial 530 ice particle size.

531 From the daily NMR diffusion experiments (using the pulse 532 sequence presented in Figure 2) we obtained the droplet size 533 distribution (DSD) for the water cores inside the hydrate shells, 534 which are presented in Figure $5 \mathrm{a}$ as a function of time post-ice535 thaw. As discussed earlier, hydrate shells have been observed in 536 the literature to form relatively quickly with a thickness of up to $537 \sim 50 \mu \mathrm{m}$. Our initial ice particles were between 150 and $250 \mu \mathrm{m}$ 538 in diameter, thus we would expect that the initial water core 539 size distribution following initial hydrate shell formation would 540 approximately be between 50 and $150 \mu \mathrm{m}$ (a total reduction in 541 diameter of $100 \mu \mathrm{m}$ ). This size range is broadly what is 542 observed in Figure 5a for the first day. On day 1, a second peak 543 in the DSD distribution is also observed at in excess of 400 $544 \mu \mathrm{m}$-this we attribute to free water, since $400 \mu \mathrm{m}$ is beyond 545 the upper detection limit of the method. This small free water 546 peak was consistently observed in repeat experiments but 547 always disappeared by day 2 , presumably having been 548 converted into hydrates. There is a significant decrease in 549 water core diameter between days 1 and 2 followed by a much 550 more gradual reduction over subsequent days. This rapid initial 551 consumption is consistent with results from previous studies for 552 other hydrate systems (see, e.g., refs 26, 62, and 65).

553 From these distributions we extracted the mean values for 554 the water core sizes for each day; the results are plotted in 555 Figure 5b. An initial rapid reduction in the mean core size is 556 observed followed by a very subtle reduction. There appears to 557 be a steady-state value around $30-40 \mu \mathrm{m}$ (corresponding to a 558 hydrate shell thickness between 55 and $110 \mu \mathrm{m}$ ) over the time 559 scale studied. From the $T_{2}$ relaxation experiments we were also 560 able to obtain the mean droplet size using eq 3 . This required 561 multiple comparisons of the equilibrium water core size (as 562 measured using PFG techniques) with relaxation measurements 563 in order to determine the required surface relaxivity, $\rho$. A value 564 of $12 \mu \mathrm{m} / \mathrm{s}$ was produced, which is consistent with the value 15 $565 \mu \mathrm{m} / \mathrm{s}$ reported for ice-water surfaces obtained via NMR 566 studies. ${ }^{76}$ Using this value, the mean droplet size distribution is 567 plotted in Figure 5 against the PFG NMR data for two 568 independent series of experiments. The agreement between 569 these two distinct measurement protocols-PFG NMR 570 measurement of restricted self-diffusion and NMR $T_{2}$ relaxation 571 measurements - is adequate post the first day for both 572 independent sets of experiments.

573 The day 1 results for experiments 1 and 2 shown in Figure $5 b$ 574 are in disagreement; however, this is no doubt affected by the 575 comparatively rapid change occurring at this temporal point 576 relative to the temporal resolution of the overall data 577 acquisition ( $\sim 1$ day). Between experiments 1 and 2 , the 578 order of measurement (PFG and $T_{2}$ relaxation) was reversed; 579 however, the results consistently show on day 1 that the $T_{2}$ 580 prediction of water core size is significantly smaller than the 581 PFG-based measurement. The $T_{2}$ measurement is sensitive to 582 the $S / V$ ratio of the confined water core and strictly can only be 583 compared with the PFG-derived mean droplet size, by assuming that the water core is a perfect sphere. The difference 584 between the PFG-derived and $T_{2}$-relaxation-derived mean 585 droplet sizes implies this is not the case on day 1 . As discussed 586 earlier, the collective studies of Genov et al., ${ }^{62}$ Chen et al., ${ }^{63}$ and 587 Rao et al. ${ }^{65}$ of flat hydrate films or shell exteriors indicate that 588 the initial hydrate film is highly porous, with the porosity 589 reducing significantly subsequently over time. Such a porous 590 hydrate film would result in a larger $S / V$ ratio with respect to 591 the relaxation measurements and, consequently, the observed 592 smaller "apparent water core diameter" $\left(\alpha(S / V)^{-1}\right)$ measured 593 relative to the diffusion measurement. The diffusion measure- 594 ment is more immune to the actual $S / V$ ratio (provided the 595 porous hydrate film presents a sufficiently low permeability) 596 and in this case is dictated more by the actual dimension of the 597 water core within the hydrate shell. Also shown in Figure 5b by 598 the solid lines is the prediction of the evolution in droplet 599 diameter from day 2 onward based on the residual water 600 amount extracted from Figure $4 \mathrm{~b}$ as a function of time. This 601 assumes the core to be a sphere and also pivots off the diameter 602 for day 2 and hence assumes it to be correct. This is in excellent 603 agreement with the diameters measured on days 3-9. 604

Thus, the evidence suggests that the initial hydrate shell is a 605 high $S / V$ porous film. This film porosity is, we hypothesize, 606 significantly reduced by day 2 , and the assumption of a 607 spherical water core is supported by the congruence of all 608 independently measured data from this temporal point onward. 609 Future work will focus on increasing the frequency of these 610 NMR measurements (requiring the employment of a higher 611 specification high-field magnet) so as to interrogate the initial 612 shell formation more thoroughly, as well as focusing on 613 methane hydrates employing appropriate NMR-compatible 614 high-pressure apparatus.

615

\section{CONCLUSIONS}

We have shown the effectiveness of using low-field NMR to 616 measure directly the water core size enclosed within a 617 cyclopentane hydrate shell and thus deduce information 618 about the evolution of the shell morphology. This is a unique 619 capability for such opaque systems. We were also able to 620 measure the hydrate growth kinetics (water consumption) 621 consistently using NMR relaxation measurements and observed 622 a transition from heat- to mass-transfer growth control. The 623 initial hydrate shell, following 1 day of growth, was 624 approximately $50 \mu \mathrm{m}$ thick and, based on the NMR 625 measurement of its surface to volume ratio, was relatively 626 porous. From day 2, consistent data were produced across 627 multiple independent NMR measurements indicating a 628 spherical core with a very low porosity that was shrinking in 629 diameter by approximately $1 \mu \mathrm{m}$ /day.

630

\section{AUTHOR INFORMATION}

\section{Corresponding Author}

*:E-mail: michael.johns@uwa.edu.au.

Notes

The authors declare no competing financial interest. DP130101461 is acknowledged. 


\section{REFERENCES}

640 (1) Sloan, E. D., Jr.; Koh, C. Clathrate hydrates of natural gases, 3rd 641 ed.; CRC press: Boca Raton, FL, USA, 2008.

642 (2) Chatti, I.; Delahaye, A.; Fournaison, L.; Petitet, J.-P. Benefits and 643 drawbacks of clathrate hydrates: A review of their areas of interest. 644 Energy Convers. Manage. 2005, 46 (9), 1333-1343.

645 (3) Hammerschmidt, E. Formation of gas hydrates in natural gas 646 transmission lines. Ind. Eng. Chem. 1934, 26 (8), 851-855.

647 (4) Sloan, E. D. A changing hydrate paradigm-From apprehension 648 to avoidance to risk management. Fluid Phase Equilib. 2005, 228, 6764974.

650 (5) Sloan, D.; Koh, C. A.; Sum, A. Natural gas hydrates in flow 651 assurance; Gulf Professional: Burlington, MA, USA, 2010.

652 (6) Turner, D. J. Clathrate Hydrate Formation in Water-in-Oil 653 Dispersions, Ph.D. Thesis; Chemical Engineering, Colorado School of 654 Mines: Golden, CO, USA, 2006.

655 (7) Turner, D. J.; Miller, K. T.; Sloan, E. D. Methane hydrate 656 formation and an inward growing shell model in water-in-oil 657 dispersions. Chem. Eng. Sci. 2009, 64 (18), 3996-4004.

658 (8) Sjoblom, J. Emulsions and Emulsion Stability: Surfactant Science 659 Series/61; CRC Press: Boca Raton, FL, USA, 2012.

660 (9) Aman, Z. M.; Olcott, K.; Pfeiffer, K.; Sloan, E. D.; Sum, A. K.; $661 \mathrm{Koh}, \mathrm{C}$. A. Surfactant adsorption and interfacial tension investigations 662 on cyclopentane hydrate. Langmuir 2013, 29 (8), 2676-2682.

663 (10) Koh, C. A.; Sloan, E. D.; Sum, A. K.; Wu, D. T. Fundamentals 664 and applications of gas hydrates. Annu. Rev. Chem. Biomol. Eng. 2011, $6652,237-257$.

666 (11) Cowie, J.; Bollavaram, P.; Erdogmus, M.; Johnson, T.; Shero, W. 667 Optimal hydrate management and new challenges in GoM deepwater using 668 "Best in Class" technologies, OTC 17328, presented at the Offshore 669 Technology Conference, Houston, Texas, USA, May, 2-5, 2005; 670 Offshore Technology Conference: Houston, TX, USA, 2005.

671 (12) Kelland, M. A. History of the development of low dosage 672 hydrate inhibitors. Energy Fuels 2006, 20 (3), 825-847.

673 (13) Ribeiro, C. P., Jr.; Lage, P. L. C. Modelling of hydrate formation 674 kinetics: State-of-the-art and future directions. Chem. Eng. Sci. 2008, 63 675 (8), 2007-2034.

676 (14) Klapproth, A.; Goreshnik, E.; Staykova, D.; Klein, H.; Kuhs, W. 677 F. Structural studies of gas hydrates. Can. J. Phys. 2003, 81 (1-2), 678 503-518.

679 (15) Davies, S. R.; Sloan, E. D.; Sum, A. K.; Koh, C. A. In situ studies 680 of the mass transfer mechanism across a methane hydrate film using 681 high-resolution confocal raman spectroscopy. J. Phys. Chem. C 2009, 682114 (2), 1173-1180.

683 (16) Kerenskaya, G.; Goldschleger, I. U.; Apkarian, V. A.; Janda, K. 684 C. Spectroscopic signatures of halogens in clathrate hydrate cages. 1. 685 Bromine. J. Phys. Chem. A 2006, 110 (51), 13792-13798.

686 (17) Luzinova, Y.; Dobbs, G. T.; Raichlin, Y.; Katzir, A.; Mizaikoff, B. 687 Infrared spectroscopic monitoring of surface effects during gas hydrate 688 formation in the presence of detergents. Chem. Eng. Sci. 2011, 66 (22), 689 5497-5503.

690 (18) Sloan, E. D., Jr.; Fleyfel, F. A molecular mechanism for gas 691 hydrate nucleation from ice. AIChE J. 1991, 37 (9), 1281-1292.

692 (19) Aichele, C. P.; Chapman, W. G.; Rhyne, L. D.; Subramani, H. J.; 693 Montesi, A.; Creek, J. L.; House, W. Nuclear magnetic resonance 694 analysis of methane hydrate formation in water-in-oil emulsions. 695 Energy Fuels 2009, 23 (2), 835-841.

696 (20) Packer, K. J.; Rees, C. Pulsed NMR studies of restricted 697 diffusion. I. Droplet size distributions in emulsions. J. Colloid Interface 698 Sci. 1972, 40 (2), 206-218.

699 (21) Ling, N. N.; Haber, A.; May, E. F.; Fridjonsson, E. O.; Johns, M. $700 \mathrm{~L}$. NMR studies of emulsion microstructure approaching the phase 701 inversion point. Colloids Surf., A 2014, 462, 244-251.

702 (22) Gao, S.; Chapman, W. G.; House, W. Application of low field 703 NMR T 2 measurements to clathrate hydrates. J. Magn. Reson. 2009, 704197 (2), 208-212.

705 (23) Melnikov, V.; Nesterov, A.; Podenko, L.; Reshetnikov, A.; 706 Shalamov, V. NMR evidence of supercooled water formation during gas hydrate dissociation below the melting point of ice. Chem. Eng. Sci. 707 2012, 71, 573-577.

(24) Majid, A. A.; Saidian, M.; Prasad, M.; Koh, C., Measurement of 709 the Water Droplet Size in Water-in-Oil Emulsions Using Low Field 710 Nuclear Magnetic Resonance for Gas Hydrate Slurry Application. Can. 711 J. Chem. 2015, DOI: 10.1139/cjc-2014-0608.

(25) Chen, L.; Sloan, E. D.; Koh, C. A.; Sum, A. K. Methane hydrate 713 formation and dissociation on suspended gas bubbles in water. J. 714 Chem. Eng. Data 2013, 59 (4), 1045-1051.

(26) Taylor, C. J.; Miller, K. T.; Koh, C. A.; Sloan, E. D., Jr. 716 Macroscopic investigation of hydrate film growth at the hydrocarbon/ 717 water interface. Chem. Eng. Sci. 2007, 62 (23), 6524-6533. 718

(27) Makogon, Y.; Mokogon, T.; Holditch, S. Several aspects of the 719 kinetics and morphology of gas hydrates, Proceedings of the Japan 720 National Oil Conference; 1998; pp 259-267.

(28) Li, S.-L.; Sun, C.-Y.; Liu, B.; Feng, X.-J.; Li, F.-G.; Chen, L.-T.; 722 Chen, G.-J. Initial thickness measurements and insights into crystal 723 growth of methane hydrate film. AIChE J. 2013, 59 (6), 2145-2154. 724

(29) Wu, R.; Kozielski, K. A.; Hartley, P. G.; May, E. F.; Boxall, J.; 725 Maeda, N. Methane-propane mixed gas hydrate film growth on the 726 surface of water and Luvicap EG solutions. Energy Fuels 2013, 27 (5), 727 $2548-2554$.

(30) Levitt, M. H. Spin dynamics. Basics of nuclear magnetic resonance; 729 John Wiley \\& Sons: Hoboken, NJ, USA, 2008.

(31) Traficante, D. D. Relaxation: An introduction. eMagRes. 1996, 731 DOI: $10.1002 / 9780470034590$.emrstm0452.

(32) Barrie, P. J. Characterization of porous media using NMR 733 methods. Annu. Rep. NMR Spectrosc. 2000, 41, 265-318. 734

(33) Kimmich, R.; Anoardo, E. Field-cycling NMR relaxometry. Prog. 735 Nucl. Magn. Reson. Spectrosc. 2004, 44 (3), 257-320. 736

(34) Brownstein, K. R.; Tarr, C. E. Spin-lattice relaxation in a system 737 governed by diffusion. J. Magn. Reson. 1977, 26 (1), 17-24. 738

(35) Brownstein, K. R.; Tarr, C. E. Diffusional theory for proton 739 spin-lattice relaxation in biological cells. Bull. Am. Phys. Soc. 1979, 24740 (3), 346.

741

(36) Casanova, F.; Perlo, J.; Blümich, B. Single-sided NMR; Springer: 742 Berlin/Heidelberg, 2011.

(37) Mitchell, J.; Chandrasekera, T. Understanding generalized 744 inversions of nuclear magnetic resonance transverse relaxation time in 745 porous media. J. Chem. Phys. 2014, 141 (22), No. 224201.

(38) Stejskal, E. O.; Tanner, J. E. Spin Diffusion Measurements: Spin 747 Echoes in the Presence of a Time-Dependent Field Gradient. J. Chem. 748 Phys. 1965, 42 (1), 288-292. 749

(39) Murday, J. S.; Cotts, R. M. Self-Diffusion Coefficient of Liquid 750 Lithium. J. Chem. Phys. 1968, 48 (11), 4938-4945. 751

(40) Grebenkov, D. S. Laplacian eigenfunctions in NMR. I. A 752 numerical tool. Concepts Magn. Reson., Part A 2008, 32A (4), 277-301. 753

(41) Lingwood, I. A.; Chandrasekera, T. C.; Kolz, J.; Fridjonsson, E. 754 O.; Johns, M. L. Emulsion droplet sizing using low-field NMR with 755 chemical shift resolution and the block gradient pulse method. J. Magn. 756 Reson. 2012, 214, 281-288.

(42) Caprihan, A.; Wang, L. Z.; Fukushima, E. A Multiple-Narrow- 758 Pulse Approximation for Restricted Diffusion in a Time-Varying Field 759 Gradient. J. Magn. Reson., Ser. A 1996, 118 (1), 94-102. 760

(43) Callaghan, P. T. A Simple Matrix Formalism for Spin Echo 761 Analysis of Restricted Diffusion under Generalized Gradient Wave- 762 forms. J. Magn. Reson. 1997, 129 (1), 74-84.

(44) Ryland, B. N.; Callaghan, P. T. Spin Echo Analysis of Restricted 764 Diffusion under Generalized Gradient Waveforms for Spherical Pores 765 with Relaxivity and Interconnections. Isr. J. Chem. 2003, 43 (1-2), 1- 766 7.

(45) Barzykin, A. V. Theory of Spin Echo in Restricted Geometries 768 under a Step-wise Gradient Pulse Sequence. J. Magn. Reson. 1999, 139769 (2), 342-353.

(46) Grebenkov, D. S. NMR survey of reflected Brownian motion. 771 Rev. Mod. Phys. 2007, 79, 1077-1137.

(47) Hollingsworth, K. G.; Johns, M. L. Measurement of emulsion 773 droplet sizes using PFG NMR and regularization methods. J. Colloid 774 Interface Sci. 2003, 258 (2), 383-389.
775 
776 (48) Sloan, E. D., Jr. Fundamental principles and applications of 777 natural gas hydrates. Nature 2003, 426 (6964), 353-363.

778 (49) Nakajima, M.; Ohmura, R.; Mori, Y. H. Clathrate hydrate 779 formation from cyclopentane-in-water emulsions. Ind. Eng. Chem. Res. 780 2008, 47 (22), 8933-8939.

781 (50) Nicholas, J. W.; Dieker, L. E.; Sloan, E. D.; Koh, C. A. Assessing 782 the feasibility of hydrate deposition on pipeline walls-Adhesion force 783 measurements of clathrate hydrate particles on carbon steel. J. Colloid 784 Interface Sci. 2009, 331 (2), 322-328.

785 (51) Barrer, R.; Edge, A. Gas hydrates containing argon, krypton and 786 xenon: kinetics and energetics of formation and equilibria. Proc. R. Soc. 787 London, Ser. A 1967, 300 (1460), 1-24.

788 (52) Wu, R.; Kozielski, K. A.; Hartley, P. G.; May, E. F.; Boxall, J.; 789 Maeda, N. Probability distributions of gas hydrate formation. AIChE J. 790 2013, 59 (7), 2640-2646.

791 (53) Lee, S.; Zhang, J.; Mehta, R.; Woo, T.-K.; Lee, J. W. Methane 792 hydrate equilibrium and formation kinetics in the presence of an 793 anionic surfactant. J. Phys. Chem. C 2007, 111 (12), 4734-4739.

794 (54) Sloan, E.; Subramanian, S.; Matthews, P.; Lederhos, J.; Khokhar, 795 A. Quantifying hydrate formation and kinetic inhibition. Ind. Eng. 796 Chem. Res. 1998, 37 (8), 3124-3132.

797 (55) Zylyftari, G.; Ahuja, A.; Morris, J. F. Nucleation of cyclopentane 798 hydrate by ice studied by morphology and rheology. Chem. Eng. Sci. 799 2014, 116, 497-507.

800 (56) Vysniauskas, A.; Bishnoi, P. A kinetic study of methane hydrate 801 formation. Chem. Eng. Sci. 1983, 38 (7), 1061-1072.

802 (57) Moon, C.; Taylor, P. C.; Rodger, P. M. Molecular dynamics 803 study of gas hydrate formation. J. Am. Chem. Soc. 2003, 125 (16), 804 4706-4707.

805 (58) Karanjkar, P. U.; Lee, J. W.; Morris, J. F. Surfactant effects on 806 hydrate crystallization at the water-oil interface: Hollow-conical 807 crystals. Cryst. Growth Des. 2012, 12 (8), 3817-3824.

808 (59) Uchida, T.; Ebinuma, T.; Kawabata, J. i.; Narita, H. Microscopic 809 observations of formation processes of clathrate-hydrate films at an 810 interface between water and carbon dioxide. J. Cryst. Growth 1999, 204 811 (3), 348-356.

812 (60) Mori, Y. H. Estimating the thickness of hydrate films from their 813 lateral growth rates: Application of a simplified heat transfer model. $J$. 814 Cryst. Growth 2001, 223 (1-2), 206-212.

815 (61) Ohmura, R.; Ogawa, M.; Yasuoka, K.; Mori, Y. H. Statistical 816 study of clathrate-hydrate nucleation in a water/hydrochlorofluor817 ocarbon system: Search for the nature of the "memory effect. J. Phys. 818 Chem. B 2003, 107 (22), 5289-5293.

819 (62) Genov, G.; Kuhs, W. F.; Staykova, D. K.; Goreshnik, E.; 820 Salamatin, A. N. Experimental studies on the formation of porous gas 821 hydrates. Am. Mineral. 2004, 89, 1228-1239.

822 (63) Chen, L.; Sloan, E. D.; Koh, C. A.; Sum, A. K. Methane Hydrate 823 Formation and Dissociation on Suspended Gas Bubbles in Water. J. 824 Chem. Eng. Data 2013, 59 (4), 1045-1051.

825 (64) Sun, C.-Y.; Chen, G.-J.; Ma, C.-F.; Huang, Q.; Luo, H.; Li, Q.-P. 826 The growth kinetics of hydrate film on the surface of gas bubble 827 suspended in water or aqueous surfactant solution. J. Cryst. Growth 828 2007, 306 (2), 491-499.

829 (65) Rao, I.; Koh, C. A.; Sloan, E. D.; Sum, A. K. Gas Hydrate 830 Deposition on a Cold Surface in Water-Saturated Gas Systems. Ind. 831 Eng. Chem. Res. 2013, 52 (18), 6262-6269.

832 (66) Hwang, M.; Wright, D.; Kapur, A.; Holder, G. An experimental 833 study of crystallization and crystal growth of methane hydrates from 834 melting ice. J. Inclusion Phenom. Mol. Recognit. Chem. 1990, 8 (1-2), $835103-116$.

836 (67) Stern, L. A.; Hogenboom, D. L.; Durham, W. B.; Kirby, S. H.; 837 Chou, I.-M. Optical-cell evidence for superheated ice under gas838 hydrate-forming conditions. J. Phys. Chem. B 1998, 102 (15), 26278392632.

840 (68) Kini, R. A.; Dec, S. F.; Sloan, E. D., Jr. Methane + propane 841 structure II hydrate formation kinetics. J. Phys. Chem. A 2004, 108 842 (44), 9550-9556.

843 (69) Hirai, S.; Okazaki, K.; Araki, N.; Yazawa, H.; Ito, H.; Hijikata, K. 844 Transport phenomena of liquid $\mathrm{CO}_{2}$ in pressurized water flow with clathrate-hydrate at the interface. Energy Convers. Manage. 1996, 37845 (6), 1073-1078.

(70) Bruker. http://www.bruker.com/products/mr/td-nmr/ 847 minispec-mq-series/mq-droplet-size-analyzer/overview.html. 2014. $\quad 848$

(71) Carr, H. Y.; Purcell, E. M. Effects of diffusion on free precession 849 in nuclear magnetic resonance experiments. Phys. Rev. 1954, 94, 630- 850 638.

(72) Meiboom, S.; Gill, D. Modified spin-echo method for measuring 852 nuclear relaxation times. Rev. Sci. Instrum. 1958, 29 (8), 688-691. 853

(73) Fridjonsson, E. O.; Graham, B. F.; Akhfash, M.; May, E. F.; 854 Johns, M. L. Optimized Droplet Sizing of Water-in-Crude Oil 855 Emulsions Using Nuclear Magnetic Resonance. Energy Fuels 2014, 856 28 (3), 1756-1764.

(74) Gogolashvili, E.; Dzjubenko, N.; Kuz'mina, N.; Margulis, B. Y.; 858 Martynenko, L. New paramagnetic relaxation reagent for water-in-oil 859 emulsions. Magn. Reson. Imaging 1994, 12 (2), 299-300.

(75) Caravan, P.; Ellison, J. J.; McMurry, T. J.; Lauffer, R. B. 861 Gadolinium (III) chelates as MRI contrast agents: structure, dynamics, 862 and applications. Chem. Rev. 1999, 99 (9), 2293-2352.

(76) Brown, J. R.; Seymour, J. D.; Brox, T. I.; Skidmore, M. L.; Wang, 864 C.; Christner, B. C.; Luo, B.-H.; Codd, S. L. Recrystallization 865 inhibition in ice due to ice binding protein activity detected by nuclear 866 magnetic resonance. Biotechnol. Rep. 2014, 3, 60-64. 